\title{
Scaling Laws for Cooperative Node Localization in Non-Line-of-Sight Wireless Networks
}

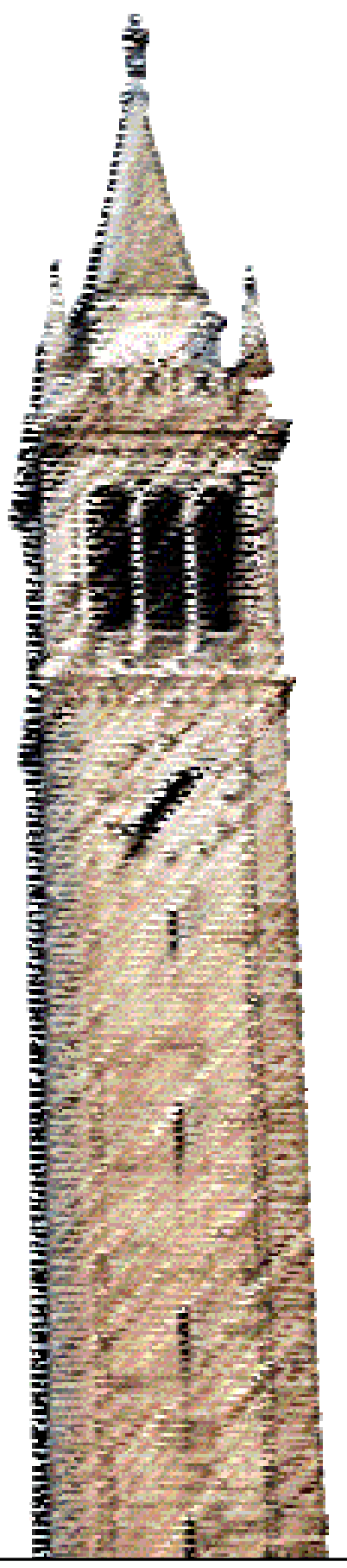

\author{
Venkatesan N E \\ Kannan Ramchandran \\ Raja Sengupta
}

Electrical Engineering and Computer Sciences University of California at Berkeley

Technical Report No. UCB/EECS-2011-20

http://www.eecs.berkeley.edu/Pubs/TechRpts/2011/EECS-2011-20.html

March 20, 2011 
Copyright (C) 2011, by the author(s).

All rights reserved.

Permission to make digital or hard copies of all or part of this work for personal or classroom use is granted without fee provided that copies are not made or distributed for profit or commercial advantage and that copies bear this notice and the full citation on the first page. To copy otherwise, to republish, to post on servers or to redistribute to lists, requires prior specific permission. 


\title{
Scaling Laws for Cooperative Node Localization in Non-Line-of-Sight Wireless Networks
}

\author{
Venkatesan. N. Ekambaram*, Kannan Ramchandran* and Raja Sengupta** \\ *Department of EECS, University of California, Berkeley \\ **Department of CEE, University of California, Berkeley \\ Email: \{venkyne,kannanr\}@eecs.berkeley.edu, sengupta@ce.berkeley.edu
}

\begin{abstract}
We study the problem of cooperative node localization in non-line-of-sight (NLOS) wireless networks and address design questions such as, "How many anchors and what fraction of line-of-sight (LOS) measurements are needed achieve a specified target accuracy?". We analytically characterize the performance improvement in localization accuracy as a function of the number of nodes in the network and the fraction of LOS measurements. In particular, we show that the CramerRao Lower Bound (CRLB) can be expressed as a product of two factors - a scalar function that depends only on the parameters of the noise distribution and a matrix that depends only on the geometry of node locations. This holds for arbitrary distance and angle measurement modalities under an additive noise model. Further, a simplified expression is obtained for the CRLB, which provides an insightful understanding of the bound and helps deduce the scaling behavior of the estimation error as a function of the number of agents and anchors in the network. The mean squared error in localization is shown to have an inverse linear relationship with the number of anchors or agents. The error is also shown to have an approximately inverse linear relationship with the fraction of LOS readings except at the extremes. The behavior at the extremes suggests that even a small fraction of LOS measurements can provide significant improvements. Conversely, a small fraction of NLOS measurements can significantly degrade the performance. Keywords- NLOS localization, Cramer-Rao Bound.
\end{abstract}

\section{INTRODUCTION}

Accurate localization of nodes is critical in applications such as vehicle safety [2], autonomous robotic systems [6], Unmanned Air Vehicle (UAV) systems, surveillance sensor networks, etc. Standard GPS receivers can have errors of over fifty meters which is unacceptable for many of these applications. The principal problem is multipath ${ }^{1}$ interference prevalent in "urban canyon" and indoor environments that introduces large bias errors in the measurements. Cooperative localization algorithms that employ a "peer-to-peer" architecture, where nodes collaboratively discard the multipath corrupted measurements and refine their position estimates, have been proposed in [18], [5] and this continues to be an active area of research. The fundamental insight in these algorithms is that, some fraction of the measurements will be produced by line-of-sight (LOS) dominated signals, and hence be fairly accurate, while some fraction will be corrupted by dominated non-line-of-sight (NLOS) reflected waves. Receivers do not know a priori which measurements are LOS and which are

\footnotetext{
${ }^{1}$ Multiple delayed versions of the same transmitted signal are received at the receiver due to reflections from different scatterers in the environment.
}

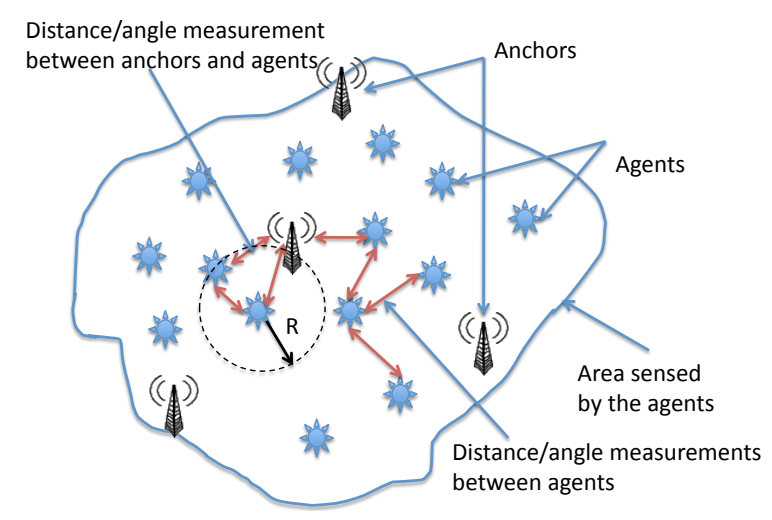

Figure 1. Example of a static sensor network.

NLOS. Hence, the task of the users is to cooperatively discard the NLOS signals, enabling them to compute high-precision position estimates. Thus the system design largely depends on the available fraction of LOS measurements. The question of how the performance behavior scales with the fraction of LOS measurements is of significant practical interest. However, there is very little work on analytical results for the NLOS case in the literature that address this question.

Further, most of the localization techniques exploit the availability of special nodes in the network designated as "anchors" with known locations and estimate the positions of "agents" or nodes with unknown locations. Examples of anchors include fixed base stations, GPS satellites, road side infrastructure for vehicular networks etc. Examples of agents include sensor nodes, robots, vehicles, UAV's etc. To achieve a target localization accuracy for different applications, the design question of how many anchors to deploy and where to deploy them still remains open. Most of the existing literature focus on developing algorithms for LOS localization, and present simulation results to analyze their performance as a function of the number of anchors in the network. One of earliest studies in this direction is that of Langendoen et al. [7], who provide a quantitative comparison of existing localization algorithms and their performance behavior as a function of the number of anchors. There has been more recent work on localization algorithms and their performance analysis which we will not detail here given that our focus is more on understanding fundamental limits.

To determine the fundamental limits of localization accuracy, the Cramer-Rao Lower Bound (CRLB), a lower bound 
on the best possible mean square error achievable using an unbiased estimator, has been analyzed under different assumptions in the literature ${ }^{2}$. Savvides et al. [12], Patwari et al. [9], Botteron et al. [1] derive expressions for the CRLB for time of arrival and angle of arrival measurements in a LOS setting and provide simulation studies to understand its behavior as a function of the number of anchors. Chang et al. [3], Weiss et al. [15], analyze the performance improvement for LOS localization when an additional anchor is introduced in the network and derive constraints on the anchor placement to obtain a performance improvement. However, the complex nature of the expressions renders it difficult to gain insights on its scaling behavior as a function of the number of nodes. The behavior has been characterized in special cases such as non-cooperative [14] and wideband localization [13].

For the NLOS setting, Qi et al. [10] and Shen et al. [13] derive the CRLB for Time of Arrival measurements, by treating the NLOS biases as unknown parameters. The results of Qi et al. [10] suggest that the NLOS measurements be detected and discarded. The derived bounds further require that the user has prior knowledge of which measurements are NLOS. In practice, one usually has prior knowledge of the NLOS noise distribution and not the nature of a reading as being LOS/NLOS. For example, exponential noise models for NLOS have been proposed in the literature [11]. An analytical characterization of the performance behavior as a function of the NLOS noise distribution is missing in the literature, which we address in this work.

We derive the CRLB for a generalized distance/angle measurement model with additive noise. We show that the CRLB can be expressed as the product of a scalar function of the noise distribution and a matrix that depends only on the node geometry and the measurement model. The scalar function provides insights into the behavior of the bound as a function of the parameters of the noise distribution and in particular, the fraction of LOS readings. To remove the dependency on the node geometry, we derive a considerably simplified expression for the sum mean squared error as a function of the newly added anchors for a distance measurement model. The localization error is shown to have an inverse linear relationship with the number of anchors, which analytically justifies the simulations of Savvides et al. [12]. A similar analysis is carried out for agents to emphasize on the benefits of cooperation.

\section{Problem Setup}

Consider a static placement of $N$ agents and $M$ anchors in a two-dimensional region of unit area (see Fig. 1). Each node obtains distance/angle measurements with respect to neighboring nodes within a communication radius $R$. Node locations will be represented by complex numbers, where the real part represents the $x$-coordinate and the imaginary part represents the $y$-coordinate. Let $u_{i}$ denote the location

\footnotetext{
${ }^{2}$ The CRLB is not a good indicator of the estimator performance at low signal-to-noise ratio [17] and for certain node geometries [4]. However, we shall not consider this in our work for now.
}

of the $i$ th agent and $v_{i}$ the location of the $i$ th anchor node. Let $\hat{r}\left(u_{i}, u_{j}\right)=r\left(u_{i}, u_{j}\right)+n_{i j}$, be the sensor measurement between nodes $u_{i}$ and $u_{j} . r\left(u_{i}, u_{j}\right)$ is some function of the node locations that depends on the measurement modality. For example, $r\left(u_{i}, u_{j}\right)=\left\|u_{i}-u_{j}\right\|$ in the case of Time of Arrival/ Time Difference of Arrival based sensors, $r\left(u_{i}, u_{j}\right) \propto \frac{1}{\left\|u_{i}-u_{j}\right\|^{\gamma}}$ for the case of Received Signal Strength measurements, $r\left(u_{i}, u_{j}\right)=\tan ^{-1} \frac{\operatorname{Im}\left(u_{i}-u_{j}\right)}{\operatorname{Re}\left(u_{i}-u_{j}\right)}$ for Angle of Arrival measurements etc.

The noise in the measurements $n_{i j}$ has a probability density function $p_{N O I S E}\left(n_{i j}\right)$. For the case of LOS measurements, $p_{N O I S E}($.$) is usually modeled as a gaussian distribution. In$ the NLOS setup, $p_{N O I S E}($.$) is typically modeled as a mixture$ distribution $\left(\alpha p_{L O S}+(1-\alpha) p_{N L O S}\right)$, where $p_{L O S}$ is taken to be zero-mean gaussian. The NLOS distribution $p_{N L O S}$, is usually modeled as ex-gaussian (sum of a gaussian and an exponential random variable) or a gaussian with a positive mean for distance measurements and uniform in the case of angle measurements. The noise parameters are assumed to be independent of the node locations in this model. The assumption is reasonable for applications with a large node density and small communication radius [2].

Let $\mathbf{u}$ be the vector of all node locations, $\hat{\mathbf{r}}$ the vector of inter-node sensor measurements and $\hat{\mathbf{u}}$ be an estimate of the node locations. We are interested in analyzing the behavior of the sum mean square error, $\sum_{i=1}^{N}\left\|u_{i}-\hat{u}_{i}\right\|^{2}$, as a function of the number of nodes and the fraction of LOS measurements. We will analyze the behavior of the CRLB, which is a lower bound on the estimation error for the class of unbiased estimators (i.e. $\mathbb{E}(\hat{\mathbf{u}})=\mathbf{u}$, where $\mathbb{E}$ is the expectation operator). Let $\eta=\left[\begin{array}{c}\mathbf{u}_{R} \\ \mathbf{u}_{I}\end{array}\right]$, where $\mathbf{u}_{\mathbf{R}}=\operatorname{Re}\{\mathbf{u}\}, \mathbf{u}_{\mathbf{I}}=\operatorname{Im}\{\mathbf{u}\}$, be the vector of parameters to be estimated. The Cramer-Rao theorem states that,

$$
\mathbb{E}\left[(\mathbf{u}-\hat{\mathbf{u}})(\mathbf{u}-\hat{\mathbf{u}})^{*}\right] \succeq F^{-1}
$$

where $\mathbf{a}^{*}$ represents conjugate transpose of a complex column vector a and the matrix $F$, known as the Fisher Information Matrix, is defined by,

$$
F_{i j} \triangleq \mathbb{E}\left\{\frac{\partial \ln p(\hat{\mathbf{r}} \mid \eta)}{\partial \eta_{i}} \frac{\partial \ln p(\hat{\mathbf{r}} \mid \eta)}{\partial \eta_{j}}\right\}
$$

The matrix inequality $A \succeq B$ is understood to mean that $A-$ $B$ is positive semi-definite. The next section deals with the derivation of the CRLB for the NLOS setting .

\section{RESULTS}

\section{A. CRLB for NLOS localization}

The following theorem provides a simplified expression for the Fisher Information Matrix.

Theorem 1. The Fisher Information Matrix can be written as $F=g\left(p_{N O I S E}\right) F_{G}$, where the matrix $F_{G}$ depends only on 
the node locations and the scalar function $g($.$) is given by,$

$$
g\left(p_{N O I S E}\right)=\mathbb{E}\left\{\left(\frac{\partial}{\partial z} \ln p_{N O I S E}(z)\right)^{2}\right\}
$$

under the assumption that $p_{\text {NOISE }}$ is differentiable over its support $[L L, U L]$ and $p(U L)-p(L L)=0$.

Proof: Appendix A.

\section{Remarks:}

- The effect of the number of nodes and the fraction of LOS readings, on the mean squared error, can be analyzed separately by studying the behavior of $F_{G}$ and $g($.$) respectively. A simplified expression for the trace$ of $F_{G}^{-1}$ is derived in the next section that details the scaling behavior of the mean squared estimation error as a function of the number of nodes.

- The scalar function $g($.$) depends only on the noise distri-$ bution and hence can be analyzed offline. For example, in the case of a mixture distribution we have,

$g\left(p_{N O I S E}\right)=\int_{-\infty}^{+\infty} \frac{\left(\alpha p_{L O S}^{\prime}(z)+(1-\alpha) p_{N L O S}^{\prime}(z)\right)^{2}}{\left(\alpha p_{L O S}(z)+(1-\alpha) p_{N L O S}(z)\right)} d z$

Setting $\alpha=1$ and $p_{L O S}(z) \sim \mathcal{N}\left(0, \sigma^{2}\right)$, we get $g\left(p_{\text {NOISE }}\right)=\frac{1}{\sigma^{2}}$, which matches with the LOS results in literature [15]. The expression for $g($.$) looks like a$ Fisher Information term, that quantifies the contribution of the noise uncertainty to the mean squared estimation error.

- The assumption on $p_{N O I S E}$ holds for a wide class of distributions such as ex-gaussian, gaussian mixtures, uniform distribution etc, that are commonly used models in the NLOS setup.

The behavior of the bound as a function of the fraction of LOS measurements $\alpha$, and the mean of the NLOS noise, is discussed in the simulations section.

\section{B. CRLB scaling as a function of the number of nodes}

In this section, we analyze the behavior of the trace of the inverse Fisher Information Matrix i.e. a lower bound on the sum mean squared error, as a function of the number of nodes in the network. We focus on the case when $r\left(u_{i}, u_{j}\right)=$ $\left\|u_{i}-u_{j}\right\|$. We undertake an incremental analysis by starting out with an existing network of $\mathrm{N}$ agents and $\mathrm{M}$ anchors and quantifying the effect of adding additional anchors and agents on the localization error. Traditionally, two classes of networks [8] have been considered while deriving scaling laws - dense networks and extended networks. Dense networks have a fixed area of node deployment and communication radius and the node density increases with the addition of new nodes. Extended networks are classes of networks in which the area of node deployment grows with the addition of new nodes and the communication radius is appropriately modified to maintain connectivity. The choice of the network model depends on the application. We focus on dense networks motivated by applications such as Intelligent Transportation Systems [2], where there is significant interest in augmenting the existing network of GPS satellites with terrestrial base stations in a fixed area. We will assume that the node density is large enough and ignore any boundary effects in our derivations.

\section{CRLB as a function of the number of anchors}

Let $F$ be the Fisher Information Matrix of a network with $N$ agents and $M$ anchors deployed in a unit area. Let $M^{\prime}$ anchors be uniform randomly added to this system of nodes and additional distance measurements are obtained by agents that are within a communication radius $R$, of these new anchors. Let $F^{\prime}$ be the new Fisher Information Matrix. For large $M^{\prime}$, the following theorem establishes the behavior of the mean squared error as a function of the newly added anchors.

Theorem 2. The lower bound on the sum mean squared localization error is given by,

$$
\operatorname{Trace}\left(F^{\prime-1}\right)=\frac{1}{g\left(p_{N O I S E}\right)} \sum_{i=1}^{2 N} \frac{1}{\lambda_{i}+\frac{\rho M^{\prime}}{2}},
$$

where $\rho=\pi R^{2}$ with $\rho M^{\prime}$ being the average number of new anchor measurements of every node and $\lambda_{i}$ 's are the eigen values of $F_{G}$ (assumed to be full rank).

Proof: Appendix B.

\section{Remarks:}

- The eigen values of the Fisher Information Matrix, $\left\{\lambda_{i}\right\}_{i=1}^{2 N}$ can be interpreted as a measure of the "precision" in the agent location estimates. The factor $\frac{\rho M^{\prime}}{2}$ is the "additional precision" from the newly added anchors.

- The expression is dominated by terms containing eigen values on the order of the minimum eigen value $\left(\lambda^{m i n}\right)$. We have,

$$
\sum_{i=1}^{2 N} \frac{1}{\lambda_{i}+\frac{\rho M^{\prime}}{2}} \rightarrow O\left(\frac{1}{M^{\prime}}\right), \text { for } \rho M^{\prime}>>2 \lambda^{\text {min }} .
$$

\section{CRLB as a function of the number of agents}

Under the same setup as before, instead of adding new anchors, let $N^{\prime}$ new agents be added to the existing system of nodes. Let $F^{\prime}$ be the new Fisher Information Matrix restricted to the first $N$ agents. The behavior of the sum mean squared error as a function of the agents is given by the following theorem.

Theorem 3. The Fisher Information Matrix $F^{\prime}$ is given by,

$$
\begin{aligned}
F^{\prime}= & F+\frac{1}{g\left(p_{N O I S E}\right)} \frac{\rho N^{\prime}}{2}\left(\left(1-\frac{1}{\rho(N+M)}\right) I_{2 N}\right. \\
& \left.-\frac{1}{\rho(N+M)}\left[\begin{array}{cc}
\mathbf{1}_{N} \mathbf{1}_{N}^{T} & \mathbf{0} \\
\mathbf{0} & \mathbf{1}_{N} \mathbf{1}_{N}^{T}
\end{array}\right]\right)
\end{aligned}
$$

Proof: Appendix C.

Corollary 1. For large $N$, assuming $F$ to be full rank, the 


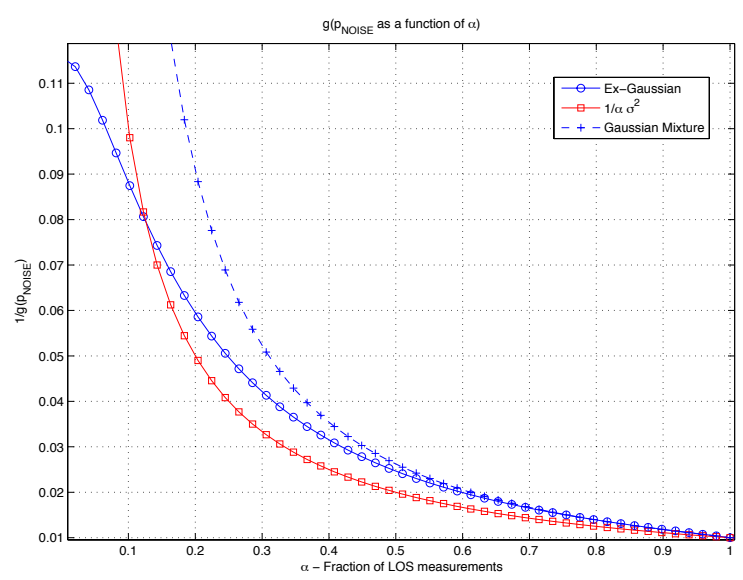

Figure 2. Variation of $\frac{1}{g\left(p_{N O I S E}\right)}$ as a function of $\alpha$ for ex-gaussian and Gaussian mixture distributions.

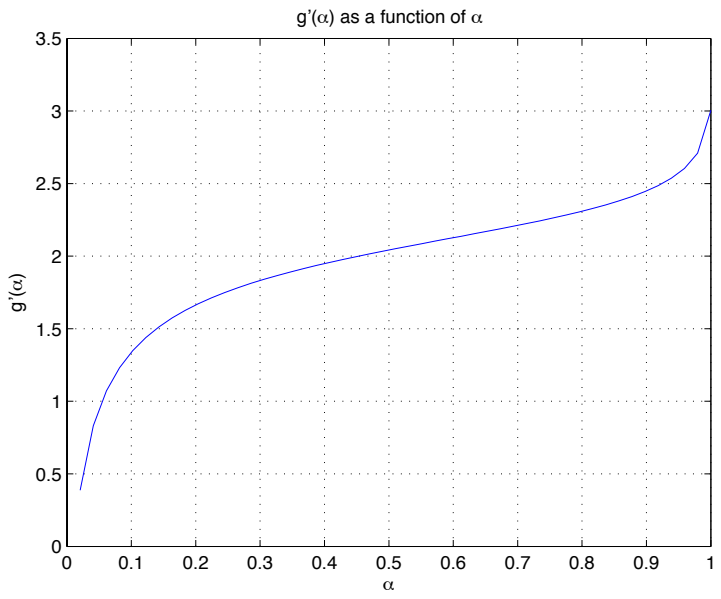

Figure 3. $g^{\prime}(\alpha)$ as a function of $\alpha$ for ex-gaussian.

above bound reduces to,

$$
\operatorname{Trace}\left(F^{\prime-1}\right)=\frac{1}{g\left(p_{N O I S E}\right)} \sum_{i=1}^{2 N} \frac{1}{\lambda_{i}+\frac{\rho N^{\prime}}{2}} .
$$

The result quantifies the benefits of co-operation between agents. The agents can be interpreted as virtual anchors in the network providing performance gains similar to that of anchors. Given the diminishing returns in accuracy with the number of anchors, it would be practically infeasible to deploy sufficient number of anchors to enable applications such as intelligent transportation systems that mandate very high accuracy requirements. However, if the vehicles were to cooperatively estimate their locations by obtaining relative distance measurements with respect to each other and utilizing measurements across time, the theorem says that one could potentially derive huge benefits in localization accuracy.

\section{Simulation RESUlts}

\section{A. CRLB scaling with the NLOS parameters}

We focus on distance measurements and consider a simple mixture model for the noise distribution. We will assume

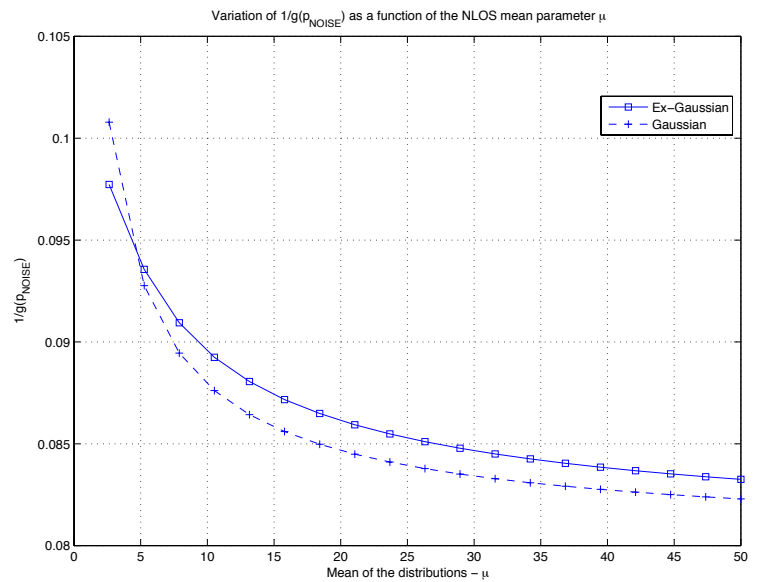

Figure 4. Variation of $\frac{1}{g\left(p_{N O I S E}\right)}$ as a function of the exponential mean parameter $\mu$ for the ex-gaussian and Gaussian mixture distributions.

that $p_{L O S} \sim \mathcal{N}\left(0, \sigma_{L O S}^{2}\right)$. Fig. 2, shows the behavior of $g\left(p_{N O I S E}\right)$ as a function of $\alpha$ for $p_{N L O S}$ being ex-gaussian and positive mean gaussian having the same mean and variance. The scaling is compared against $\frac{\sigma^{2}}{\alpha}$ which seems to be a good approximation of the behavior at higher values of $\alpha$.

Fig 3 shows a plot of $g^{\prime}(\alpha)=\frac{\partial}{\partial \alpha} g($.$) . The interesting trend$ to note here is that the increment in $g($.$) varies sharply at lower$ values and higher values of $\alpha$. This suggests that at lower values of $\alpha$, even a small fraction of LOS measurements can provide a significant decrease in the mean square error. The plot also suggests that at higher values of $\alpha$, as we transit from a LOS to NLOS regime, a small fraction of NLOS measurements can contribute to a significant degradation in the performance. The performance degradation can be attributed to the lack of knowledge of whether a particular reading is LOS or NLOS.

Fig. 4 shows the behavior of $g($.$) as a function of the$ mean of the NLOS distribution. As the mean increases, the performance improves since it is easier to identify the NLOS measurements. However the performance does not vary significantly as a function of the mean.

\section{B. CRLB scaling with the number of nodes}

Consider $N$ agents and $M$ anchors uniformly placed in a circular region of area $A$. Nodes that are within a communication radius $R$, obtain pairwise distance measurements. In the first set of simulations, $M^{\prime}$ additional anchors are uniformly placed in the same region and the CRLB is evaluated as a function of $M^{\prime}$. The scaling behavior is consistent with the theory (see Fig. 5). The CRLB is normalized with respect to the number of agents and the communication radius.

For the second set of simulations $N^{\prime}$ additional agents are uniformly deployed in the same region and the CRLB is evaluated as a function of $N^{\prime}$. The theoretical and simulated bounds are shown to match well in terms of their scaling behavior (Fig. 6). For the same initial deployment of agents and anchors, simulations were carried out by adding more anchors to the network as opposed to new agents. Fig. 6 also shows the CRLB as a function of the number of anchors. 


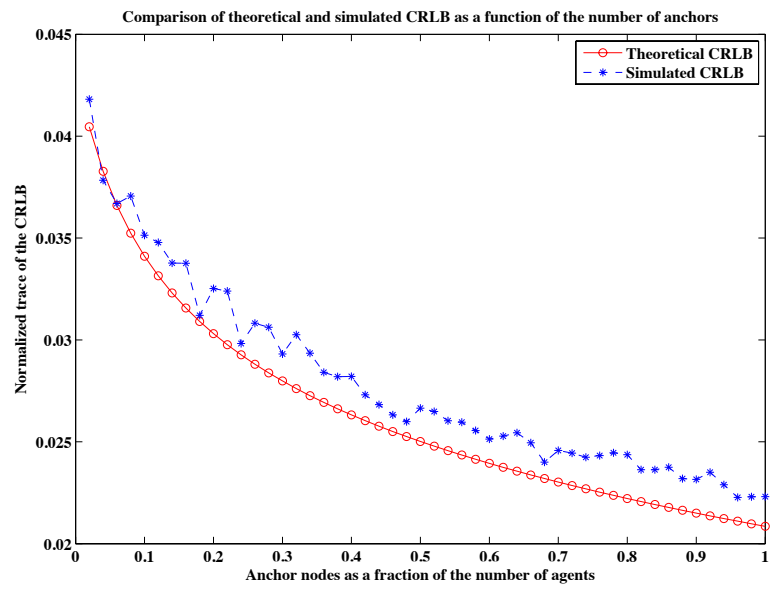

Figure 5. Comparison of the normalized theoretical and simulated CRLB as function of the number of anchor nodes. The parameters used for the simulation are $N=50, M=5, R=3, A=100, \sigma=1$.

These two bounds can be thought of as representing the two extremal cases of adding nodes, one that corresponds to adding nodes with exactly known locations (anchors) and the other corresponding to adding nodes with unknown locations (agents). For the case of adding nodes with partial location knowledge, one can expect to obtain a performance that lies in between these two extremal cases. The law of diminishing returns in the performance is clearly visible in the simulations. The gap in the bounds for all the simulations can be attributed to approximations in using the law of large numbers and boundary effects that were ignored in the derivations.

\section{CONClusion}

We focused on the problem of cooperative node localization in a NLOS wireless network and studied the behavior of the localization error as a function of the number of nodes in the network and the fraction of LOS measurements. We showed that the CRLB can be written as a product of a scalar function that depends on the parameters of the noise distribution and a matrix that depends only on the geometry of the node placement. The mean squared error was shown to have an inverse linear relationship with the number of nodes in the network and the fraction of LOS measurements.

Our work focused on providing design guidelines for number of anchors to be deployed. However the question of optimal anchor placement is still widely open. Further the uniformity assumptions in the network deployment could be restrictive in practice. The parameters of the noise distributions could also be added as parameters that need to be estimated, though the CRLB would be more complicated and harder to derive insights from. The effect of mobility on the localization performance is a future direction of research.

\section{REFERENCES}

[1] C. Botteron, A. Host-Madsen, and M. Fattouche. Cramer-Rao bounds for the estimation of multipath parameters and mobiles' positions in asynchronous DS-CDMA systems. Signal Processing, IEEE Transactions on, 52(4):862-875, 2004.

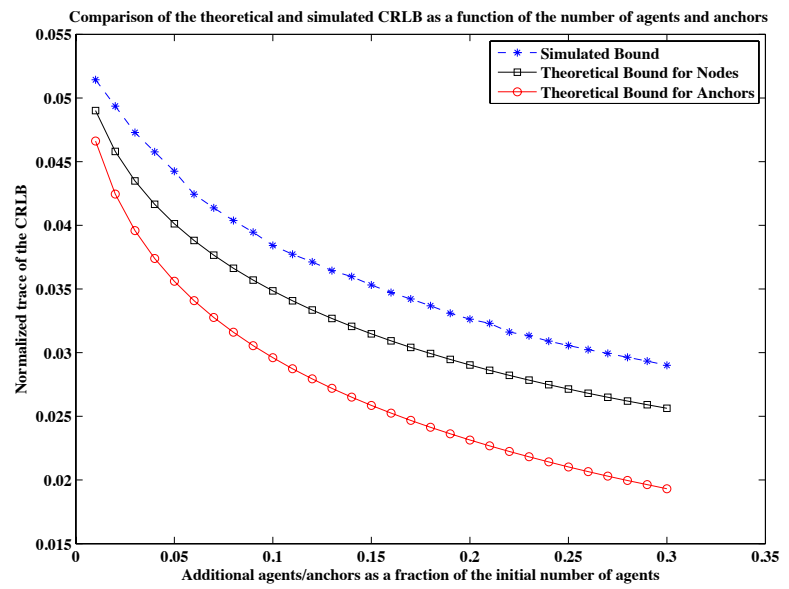

Figure 6. Comparison of the normalized theoretical and simulated CRLB as function of the number of agents. The parameters used for the simulation are $N=100, M=10, R=3, A=100, \sigma=1$.

[2] A. Boukerche, H. Oliveira, E. Nakamura, and A. Loureiro. Vehicular ad hoc networks: a new challenge for localization-based systems. Computer Communications, 31(12):2838-2849, 2008.

[3] C. Chang and A. Sahai. Estimation bounds for localization. In IEEE SECON 2004, pages 415-424, 2004.

[4] S. Dulman, P. Havinga, A. Baggio, and K. Langendoen. Revisiting the Cramer-Rao Bound for Localization Algorithms. 4th IEEE/ACM DCOSS Work-in-progress paper, 2008.

[5] V. Ekambaram and K. Ramchandran. Distributed High Accuracy Peerto-Peer Localization in Mobile Multipath Environments. In IEEE GLOBECOM, Miami, Florida, 2010.

[6] E. Kiriy and M. Buehler. Three-state extended kalman filter for mobile robot localization. McGill University., Montreal, Canada, Tech. Rep. $T R-C I M, 5,2002$

[7] K. Langendoen and N. Reijers. Distributed localization in wireless sensor networks: a quantitative comparison. Computer Networks, 43(4):499-518, 2003.

[8] A. Ozgur, O. Lévêque, and D. Tse. Hierarchical Cooperation Achieves Optimal Capacity Scaling in Ad Hoc Networks. Information Theory, IEEE Transactions on, 53(10):3549-3572, 2007.

[9] N. Patwari, J. Ash, S. Kyperountas, A. Hero III, R. Moses, and N. Correal. Locating the nodes: cooperative localization in wireless sensor networks. IEEE Signal Processing Magazine, 22(4):54-69, 2005.

[10] Y. Qi, H. Kobayashi, and H. Suda. Analysis of wireless geolocation in a non-line-of-sight environment. IEEE Transactions on wireless communications, 5(3), 2006.

[11] A. Saleh and R. Valenzuela. A statistical model for indoor multipath propagation. Selected Areas in Communications, IEEE Journal on, 5(2):128-137, 1987.

[12] A. Savvides, W. Garber, S. Adlakha, R. Moses, and M. Srivastava. On the error characteristics of multihop node localization in ad-hoc sensor networks. In IPSN 2003, pages 317-332. Springer-Verlag, 2003.

[13] Y. Shen, H. Wymeersch, and M. Win. Fundamental limits of wideband localization-part II: Cooperative networks. IEEE Trans. Inf. Theory, 2009.

[14] M. Spirito. On the accuracy of cellular mobile station location estimation. Vehicular Technology, IEEE Trans. on, 50(3):674-685, 2002.

[15] A. Weiss and J. Picard. Improvement of Location Accuracy by Adding Nodes to Ad-Hoc Networks. Wireless Personal Communications, 44(3):283-294, 2008.

[16] A. Weiss and J. Picard. Maximum-likelihood position estimation of network nodes using range measurements. Signal Processing, IET, 2(4):394-404, 2008.

[17] A. Weiss and E. Weinstein. Composite bound on the attainable mean square error in passive time-delay estimation from ambiguity prone signals. Inf. Theory, IEEE Trans on, 28(6):977-979, 2002.

[18] H. Wymeersch, J. Lien, and M. Win. Cooperative localization in wireless networks. Proceedings of the IEEE, 97(2):427-450, 2009. 


\section{APPENDIX A}

EVALUATION OF THE CRLB

This section focuses on evaluating the different entries of the Fisher Information Matrix. For simplicity, let us focus on the case where $r\left(u_{i}, u_{j}\right)=d\left(u_{i}, u_{j}\right)=\left\|u_{i}-u_{j}\right\|$. Let $\hat{\mathbf{d}}=\hat{\mathbf{r}}$. The $(i, j)$ th entry in the matrix is given by,

$$
F_{i j} \triangleq \mathbb{E}\left\{\frac{\partial \ln p(\hat{\mathbf{d}} \mid \eta)}{\partial \eta_{i}} \frac{\partial \ln p(\hat{\mathbf{d}} \mid \eta)}{\partial \eta_{j}}\right\} .
$$

Let $\mathcal{N}(i)$ be the set of neighbors of node $i$. Lets focus on the case when $\eta_{i}=u_{R_{i}}$ and $\eta_{j}=u_{R_{j}}$. We have,

$$
\begin{aligned}
\frac{\partial \ln p(\hat{\mathbf{d}} \mid \eta)}{\partial \eta_{i}} & =\frac{\partial \ln p(\hat{\mathbf{d}} \mid \eta)}{\partial u_{R_{i}}}, \\
& =\sum_{l \in \mathcal{N}(i)} \frac{\partial \ln p\left(\hat{d}_{i l} \mid u_{i}, u_{l}\right)}{\partial u_{R_{i}}}, \\
& =\sum_{l \in \mathcal{N}(i)} \frac{\partial \ln p\left(z\left(u_{i}, u_{l}\right)\right)}{\partial z\left(u_{i}, u_{l}\right)} \frac{\partial z\left(u_{i}, u_{l}\right)}{\partial u_{R_{i}}},
\end{aligned}
$$

where $z\left(u_{i}, u_{l}\right)=\hat{d}_{i l}-\left\|u_{i}-u_{l}\right\|$. Further,

$$
\begin{aligned}
\frac{\partial z\left(u_{i}, u_{l}\right)}{\partial u_{R_{i}}} & =\frac{\left(u_{R_{i}}-u_{R_{l}}\right)}{\left\|u_{i}-u_{l}\right\|}, \\
& =\cos \left(\phi_{i l}\right),
\end{aligned}
$$

where $\phi_{i l}$ is the angle between the vector joining the nodes $(i, l)$ and the horizontal axis. Similarly we get,

$$
\frac{\partial \ln p(\hat{\mathbf{d}} \mid \eta)}{\partial u_{R_{j}}}=\sum_{m \in \mathcal{N}(j)} \frac{\partial \ln p\left(z\left(u_{j}, u_{m}\right)\right)}{\partial z\left(u_{j}, u_{m}\right)} \cos \left(\phi_{j m}\right) \text {. }
$$

Thus we have,

$$
\begin{aligned}
F_{i j} & =\mathbb{E}\left\{\sum_{l \in \mathcal{N}(i)} \frac{\partial \ln p\left(z\left(u_{i}, u_{l}\right)\right)}{\partial z\left(u_{i}, u_{l}\right)} \cos \left(\phi_{i l}\right) \sum_{m \in \mathcal{N}(j)} \frac{\partial \ln p\left(z\left(u_{j}, u_{m}\right)\right)}{\partial z\left(u_{j}, u_{m}\right)} \cos \left(\phi_{j m}\right)\right\}, \\
& =\mathbb{E}\left\{\sum_{l \in \mathcal{N}(i)} \frac{p^{\prime}\left(z\left(u_{i}, u_{l}\right)\right)}{p\left(z\left(u_{i}, u_{l}\right)\right)} \cos \left(\phi_{i l}\right) \sum_{m \in \mathcal{N}(j)} \frac{p^{\prime}\left(z\left(u_{j}, u_{m}\right)\right)}{p\left(z\left(u_{j}, u_{m}\right)\right)} \cos \left(\phi_{j m}\right)\right\}
\end{aligned}
$$

Let us simplify this for the different cases of node pairs.

Case 1: $i=j$

$$
\begin{aligned}
F_{i i} & =\mathbb{E}\left\{\sum_{l \in \mathcal{N}(i)} \cos ^{2}\left(\phi_{i l}\right)\left(\frac{p^{\prime}\left(z\left(u_{i}, u_{l}\right)\right)}{p\left(z\left(u_{i}, u_{l}\right)\right)}\right)^{2}+\sum_{(l \neq m) \in \mathcal{N}(i)} \cos \left(\phi_{i l}\right) \cos \left(\phi_{i m}\right) \frac{p^{\prime}\left(z\left(u_{i}, u_{l}\right)\right)}{p\left(z\left(u_{i}, u_{l}\right)\right)} \frac{p^{\prime}\left(z\left(u_{i}, u_{m}\right)\right)}{p\left(z\left(u_{i}, u_{m}\right)\right)}\right\}, \\
& =\sum_{l \in \mathcal{N}(i)} \cos ^{2}\left(\phi_{i l}\right) \mathbb{E}\left\{\left(\frac{p^{\prime}\left(z\left(u_{i}, u_{l}\right)\right)}{p\left(z\left(u_{i}, u_{l}\right)\right)}\right)^{2}\right\}+\sum_{(l \neq m) \in \mathcal{N}(i)} \cos \left(\phi_{i l}\right) \cos \left(\phi_{i m}\right) \mathbb{E}\left\{\frac{p^{\prime}\left(z\left(u_{i}, u_{l}\right)\right)}{p\left(z\left(u_{i}, u_{l}\right)\right)}\right\} \mathbb{E}\left\{\frac{p^{\prime}\left(z\left(u_{i}, u_{m}\right)\right)}{p\left(z\left(u_{i}, u_{m}\right)\right)}\right\}, \\
& =g\left(p_{N O I S E}\right) \sum_{l \in \mathcal{N}(i)} \cos ^{2}\left(\phi_{i l}\right),
\end{aligned}
$$


where $g\left(p_{N O I S E}\right)=\mathbb{E}\left\{\left(\frac{p^{\prime}\left(z\left(u_{i}, u_{l}\right)\right)}{p\left(z\left(u_{i}, u_{l}\right)\right)}\right)^{2}\right\}$ and $\mathbb{E}\left\{\frac{p^{\prime}\left(z\left(u_{i}, u_{l}\right)\right)}{p\left(z\left(u_{i}, u_{l}\right)\right)}\right\}=0$. Assuming $p_{\text {NOISE }}(z)$ to be differentiable on its support $[L L, U L]$ and that $p(U L)=p(L L)$, we have,

$$
\begin{aligned}
\mathbb{E}\left\{\frac{p^{\prime}\left(z\left(u_{i}, u_{l}\right)\right)}{p\left(z\left(u_{i}, u_{l}\right)\right)}\right\} & =\int_{\mathrm{LL}}^{\mathrm{UL}} \frac{\partial}{\partial z} p(z) d z \\
& =p(U L)-p(L L) \\
& =0 .
\end{aligned}
$$

Case 2: $j \in \mathcal{N}(i)$

$$
\begin{aligned}
F_{i j} & =\mathbb{E}\left\{\sum_{l \in \mathcal{N}(i)} \sum_{m \in \mathcal{N}(j)} \cos \left(\phi_{i l}\right) \cos \left(\phi_{j m}\right) \frac{p^{\prime}\left(z\left(u_{i}, u_{l}\right)\right)}{p\left(z\left(u_{i}, u_{l}\right)\right)} \frac{p^{\prime}\left(z\left(u_{j}, u_{m}\right)\right)}{p\left(z\left(u_{j}, u_{m}\right)\right)}\right\}, \\
& =g\left(p_{\text {NOISE }}\right) \cos ^{2}\left(\phi_{i j}\right) .
\end{aligned}
$$

Case 2: $j \notin \mathcal{N}(i)$

$$
\begin{aligned}
F_{i j} & =\mathbb{E}\left\{\sum_{l \in \mathcal{N}(i)} \frac{p^{\prime}\left(z\left(u_{i}, u_{l}\right)\right)}{p\left(z\left(u_{i}, u_{l}\right)\right)} \cos \left(\phi_{i l}\right)\right\} \mathbb{E}\left\{\sum_{m \in \mathcal{N}(j)} \frac{p^{\prime}\left(z\left(u_{j}, u_{m}\right)\right)}{p\left(z\left(u_{j}, u_{m}\right)\right)} \cos \left(\phi_{j m}\right)\right\}, \\
& =0 .
\end{aligned}
$$

Thus for $\eta_{i}=u_{R_{i}}$ and $\eta_{j}=u_{R_{j}}$, we get

$$
F_{i j}=g\left(p_{\text {NOISE }}\right)\left\{\begin{array}{rl}
\sum_{l \in \mathcal{N}(i)} \cos ^{2}\left(\phi_{i l}\right) & \text { if } i=j \\
\cos ^{2}\left(\phi_{i j}\right) & \text { if } j \in \mathcal{N}(i) \\
0 & \text { o.w. }
\end{array} .\right.
$$

Using similar arguments we can derive the rest of the entries of the Fisher matrix as follows.

For $\eta_{i}=u_{I_{i}}$ and $\eta_{j}=u_{I_{j}}$ we have,

$$
F_{i j}=g\left(p_{\text {NOISE }}\right)\left\{\begin{array}{cl}
\sum_{l \in \mathcal{N}(i)} \sin ^{2}\left(\phi_{i l}\right) & \text { if } i=j \\
\sin ^{2}\left(\phi_{i j}\right) & \text { if } j \in \mathcal{N}(i) \\
0 & \text { o.w. }
\end{array} .\right.
$$

For $\eta_{i}=u_{R_{i}}$ and $\eta_{j}=u_{I_{j}}$ we have,

$$
F_{i j}=g\left(p_{\text {NOISE }}\right)\left\{\begin{array}{rl}
\sum_{l \in \mathcal{N}(i)} \sin \left(\phi_{i l}\right) \cos \left(\phi_{i l}\right) & \text { if } i=j \\
\sin \left(\phi_{i j}\right) \cos \left(\phi_{i j}\right) & \text { if } j \in \mathcal{N}(i) \\
0 & \text { o.w. }
\end{array} .\right.
$$

Extracting the common scalar $g\left(p_{N O I S E}\right)$ from each term in the matrix we can express the Fisher Information Matrix as,

$$
F=g\left(p_{N O I S E}\right) F_{G},
$$

where $F_{G}$ only depends on the node geometry, the function $r(.,$.$) that depends on the sensing modality and is independent of$ the noise distribution. The CRLB is thus given by,

$$
\mathbb{E}\left[(\mathbf{u}-\hat{\mathbf{u}})(\mathbf{u}-\hat{\mathbf{u}})^{*}\right] \succeq \frac{1}{g\left(p_{N O I S E}\right)} F_{G}^{-1} .
$$




\section{APPENDIX B}

DERIVATION OF THE CRLB FOR ANCHORS

A simplified expression for the CRLB as a function of the number of anchors is derived here. Recall that $\mathbf{u}$ and $\mathbf{v}$ are the vectors of agent and anchor locations. Let $\mathbf{x}=\left[\begin{array}{l}\mathbf{u} \\ \mathbf{v}\end{array}\right] \in \mathbb{C}^{\mathbf{N}+\mathbf{M}}$ be the vector of all the node locations. The location difference, $x_{i}-x_{j}$, between any two nodes can be described by the $N \times M$ vector $\mathbf{e}_{i j}$, whose $i$ th entry is 1 and $j$ th entry is -1 and all other entries are set to zero. Thus we get

$$
\mathbf{e}_{i j}^{T} \mathbf{x}=x_{i}-x_{j} .
$$

Let $L$ be the total number of distance measurements obtained in the network. We will assume that distance measurements are obtained between nodes that are within a communication radius $R$ of each other. Collecting all the location differences we get the following relation,

$$
E \mathbf{x}=\mathbf{y},
$$

where the rows of $E \in \mathbb{R}^{L \times(N+M)}$, are the vectors $\mathbf{e}_{i j}^{T}$ and $\mathbf{y} \in \mathbb{C}^{\mathbf{L} \times \mathbf{1}}$, is a complex vector of all the available location differences of nodes that are within a radius $R$ of each other. The absolute value of each entry in the vector $\mathbf{y}$ denotes the distance between the corresponding two nodes obtained from the matrix $E$.

Let $E=\left[E_{1} E_{2}\right]$, where $E_{1} \in \mathbb{R}^{L \times N}$ and $E_{2} \in \mathbb{R}^{L \times M}$. We can then write,

$$
E_{1} \mathbf{u}+E_{2} \mathbf{v}=\mathbf{y} .
$$

Let $\mathbf{d}$ denote the vector of all distances between nodes having observations i.e. $\mathbf{d}=|\mathbf{y}|$, where $|\mathbf{y}|$ is a notation used to denote a vector whose components are the absolute values of the individual components of $\mathbf{y}$. Let $\hat{\mathbf{d}}$ denote the vector of gaussian pairwise distance measurements i.e.

$$
\hat{d}_{j}=d_{j}+n_{j}
$$

where $n_{j} \sim N\left(0, \sigma^{2}\right)$. Define the two real diagonal matrices,

$$
\begin{aligned}
D_{R} & \triangleq \operatorname{Re}\left\{\operatorname{diag}\left\{y_{1} /\left|y_{1}\right| \ldots \ldots . y_{L} /\left|y_{L}\right|\right\}\right\} \\
D_{I} & \triangleq \operatorname{Im}\left\{\operatorname{diag}\left\{y_{1} /\left|y_{1}\right| \ldots \ldots . y_{L} /\left|y_{L}\right|\right\}\right\} .
\end{aligned}
$$

The authors in [16] obtain a compact representation for the Fisher Information Matrix for LOS Gaussian noise as shown below,

$$
F=\frac{1}{\sigma^{2}}\left[\begin{array}{cc}
E_{1}^{T} D_{R}^{2} E_{1} & E_{1}^{T} D_{R} D_{I} E_{1} \\
E_{1}^{T} D_{R} D_{I} E_{1} & E_{1}^{T} D_{I}^{2} E_{1}
\end{array}\right]
$$

$F$ is assumed to be invertible. In our generalized case, this reduces to

$$
F=\frac{1}{g\left(p_{\text {NOISE }}\right)}\left[\begin{array}{cc}
E_{1}^{T} D_{R}^{2} E_{1} & E_{1}^{T} D_{R} D_{I} E_{1} \\
E_{1}^{T} D_{R} D_{I} E_{1} & E_{1}^{T} D_{I}^{2} E_{1}
\end{array}\right],
$$

We will ignore the scaling factor $\frac{1}{g\left(p_{N O I S E)}\right.}$ and work with only the matrix.

Let us suppose that we add a single anchor to the set of existing nodes. Let $l$ be the number of additional distance measurements that are obtained. Without loss of generality assume that the first $l$ nodes get measurements with respect to the new anchor. The equation relating the agent locations to the location differences, $E \mathbf{x}=\mathbf{y}$ now gets updated to

$$
\left[\begin{array}{ccc}
E_{1} & E_{2} & 0 \\
\mathbf{I}_{l} \mid \mathbf{0} & \mathbf{0} & -\mathbf{1}
\end{array}\right]\left[\begin{array}{c}
\mathbf{x} \\
x_{N+M+1}
\end{array}\right]=\left[\begin{array}{c}
\mathbf{y} \\
y_{L+1} \\
\cdot . \\
y_{L+l}
\end{array}\right] .
$$

where $\mathrm{I}_{l}$ is the identity matrix of dimension $l, \mathbf{0}$ and $\mathbf{1}$ are vectors/matrices consisting of all zeros and all ones respectively, of appropriate dimensions. Let $\Delta_{1} \triangleq\left[\mathbf{I}_{l} \mid \mathbf{0}\right]$. Define

$$
\begin{aligned}
D_{R_{1}} & \triangleq \operatorname{Re}\left\{\operatorname{diag}\left\{y_{L+1} /\left|y_{L+1}\right| \ldots \ldots . y_{L+l} /\left|y_{L+l}\right|\right\}\right\} \\
D_{I_{1}} & \triangleq \operatorname{Im}\left\{\operatorname{diag}\left\{y_{L+1} /\left|y_{L+1}\right| \ldots \ldots . y_{L+l} /\left|y_{L+l}\right|\right\}\right\}
\end{aligned}
$$


After simple block matrix multiplications the new Fisher matrix can be written as,

$$
\tilde{F}=\left[F+\left[\begin{array}{cc}
\Delta_{1}^{T} D_{R_{1}}^{2} \Delta_{1} & \Delta_{1}^{T} D_{R_{1}} D_{I_{1}} \Delta_{1} \\
\Delta_{1}^{T} D_{R_{1}} D_{I_{1}} \Delta_{1} & \Delta_{1}^{T} D_{I_{1}}^{2} \Delta_{1}
\end{array}\right]\right] .
$$

Thus, if we add $M^{\prime}$ anchors recursively in the network, the new Fisher matrix can be expressed as,

$$
\tilde{F}=F+\sum_{i=1}^{M^{\prime}}\left[\begin{array}{cc}
\Delta_{i}^{T} D_{R_{i}}^{2} \Delta_{i} & \Delta_{i}^{T} D_{R_{i}} D_{I_{i}} \Delta_{i} \\
\Delta_{i}^{T} D_{R_{i}} D_{I_{i}} \Delta_{i} & \Delta_{i}^{T} D_{I_{i}}^{2} \Delta_{i}
\end{array}\right] .
$$

Here the matrices $\Delta_{i}$ of size $N \times l_{i}$ where $l_{i}$ nodes get measurements with the $i$ th newly added anchor, have ones corresponding to the columns of the nodes with which the $i$ th newly introduced anchor gets measurements. $D_{R_{i}}, D_{I_{i}}$ have definition similar to $D_{R_{1}}$ and $D_{I_{1}}$. For simplicity lets first consider the case where the newly introduced anchors have measurements with all the agents. We then have $\Delta_{i}=I_{N}, \forall i$ giving us

$$
\tilde{F}=F+\sum_{i=1}^{M^{\prime}}\left[\begin{array}{cc}
D_{R_{i}}^{2} & D_{R_{i}} D_{I_{i}} \\
D_{R_{i}}{\stackrel{D}{I_{i}}}^{2} & D_{I_{i}}^{2}
\end{array}\right]
$$

By definition, $D_{R_{i}}(j)=\frac{\operatorname{Re}\left(y_{k}\right)}{\left|y_{k}\right|}$ where $k=L+(i-1) N+j$. This can also be equivalently written as $D_{R_{i}}(j)=\cos \left(\phi_{i j}\right)$ where $\phi_{i j}$ is the angle made by the line joining $i$ th anchor node and the $j$ th node, with the horizontal axis. Let us assume that each anchor that is newly introduced is randomly placed in the field independent of all other nodes. Thus for each node $j,\left\{\phi_{i j}\right\}_{i=1}^{M^{\prime}}$ 's are i.i.d and distributed $\mathrm{U}(0,2 \pi)$. Similarly $D_{I_{i}}(j)=\sin \left(\phi_{i j}\right)$ and by the strong law of large numbers we get,

$$
\begin{aligned}
\sum_{i} D_{R_{i}}^{2}(j) & =\sum_{i=1}^{M^{\prime}} \cos ^{2}\left(\phi_{i j}\right) \rightarrow \frac{M^{\prime}}{2} \\
\sum_{i} D_{I_{i}}^{2}(j) & =\sum_{i=1}^{M^{\prime}} \sin ^{2}\left(\phi_{i j}\right) \rightarrow \frac{M^{\prime}}{2} \\
\sum_{i} D_{I_{i}}(j) D_{R_{i}}(j) & =\sum_{i=1}^{M^{\prime}} \sin \left(\phi_{i j}\right) \cos \left(\phi_{i j}\right) \rightarrow 0
\end{aligned}
$$

The Fisher matrix now simplifies to,

$$
\tilde{F}=F+\frac{M^{\prime}}{2} I_{2 N}
$$

We know that the Fisher Information Matrix is a covariance matrix and hence is symmetric positive definite. We can write $F=U \Lambda U^{H}$, where $\Lambda$ is a diagonal matrix of the eigen values of $F$ and $U U^{H}=U^{H} U=I$. We also know that $\operatorname{Tr}(A B C)=$ $\operatorname{Tr}(C A B)=\operatorname{Tr}(B C A)$. This gives us,

$$
\begin{aligned}
\operatorname{Trace}\left(\tilde{F}^{-1}\right) & =\operatorname{Trace}\left(U \Lambda U^{H}+\frac{M^{\prime}}{2} I_{2 N}\right)^{-1} \\
& =\operatorname{Trace}\left(U\left(\Lambda+\frac{M^{\prime}}{2} I_{2 N}\right) U^{H}\right)^{-1} \\
& =\sum_{i=1}^{2 N} \frac{1}{\lambda_{i}+\frac{M^{\prime}}{2}} .
\end{aligned}
$$

It is now easy to extend the analysis to the case when each anchor node has measurements only with the nodes that are within a radius $R$. Let $A$ be the total area of the field where the nodes are placed. Let $\rho=\frac{\pi R^{2}}{A}$ and then $\rho M^{\prime}$ would be the average number of neighbors of each node. In this case we would have $\Delta_{i}^{T} D_{R_{i}}^{2} \Delta_{i} \rightarrow \frac{\rho M^{\prime}}{2} I_{N}$ and so on. Thus the analysis would go through and we get

$$
\operatorname{Trace}\left(\tilde{F}^{-1}\right)=\sum_{i=1}^{2 N} \frac{1}{\lambda_{i}+\frac{\rho M^{\prime}}{2}}
$$




\section{APPENDIX C}

\section{DERIVATION OF THE CRLB FOR AGENTS}

The setup is similar as in the previous case with $N$ agents, $M$ anchors and $L$ distance measurements. We are interesting in characterizing the behavior of the CRLB after adding $N^{\prime}$ agents to the existing network of anchors and agents. Consider the simple case when $N^{\prime}=1$. We will assume for now that the newly introduced agent has measurements with all of the existing $N$ agents and $M$ anchors. Let $\tilde{F}, \tilde{E}, \tilde{E}_{1}, \tilde{D_{R}}, \tilde{D_{I}}$, be the new set of matrices obtained after adding this node. We then have the following

$$
\begin{gathered}
\tilde{E}=\left[\begin{array}{ccc}
E_{1} & \mathbf{0}_{L} & E_{2} \\
-I_{N} & \mathbf{1}_{N} & 0 \\
\mathbf{0}_{N} & \mathbf{1}_{M} & -I_{M}
\end{array}\right], \\
\tilde{E}_{1}=\left[\begin{array}{cc}
E_{1} & \mathbf{0}_{L} \\
-I_{N} & \mathbf{1}_{N} \\
\mathbf{0}_{N} & \mathbf{1}_{M}
\end{array}\right] .
\end{gathered}
$$

Let

$$
\begin{aligned}
D_{R_{11}} \triangleq \operatorname{Re}\left\{\operatorname{diag}\left\{y_{L+1} /\left|y_{L+1}\right| \ldots \ldots y_{L+N} /\left|y_{L+N}\right|\right\}\right\} \\
D_{R_{12}} \triangleq \operatorname{Re}\left\{\operatorname{diag}\left\{y_{L+N+1} /\left|y_{L+N+1}\right| \ldots \ldots y_{L+N+M} /\left|y_{L+N+M}\right|\right\}\right\} \\
D_{I_{11}} \triangleq \operatorname{Im}\left\{\operatorname{diag}\left\{y_{L+1} /\left|y_{L+1}\right| \ldots \ldots . y_{L+N} /\left|y_{L+N}\right|\right\}\right\} \\
D_{I_{12}} \triangleq \operatorname{Im}\left\{\operatorname{diag}\left\{y_{L+N+1} /\left|y_{L+N+1}\right| \ldots \ldots y_{L+N+M} /\left|y_{L+N+M}\right|\right\}\right\}
\end{aligned}
$$

Then

$$
\begin{gathered}
\tilde{D}_{R}=\left[\begin{array}{ccc}
D_{R} & \mathbf{0} & \mathbf{0} \\
\mathbf{0} & D_{R_{11}} & \mathbf{0} \\
\mathbf{0} & \mathbf{0} & D_{R_{12}}
\end{array}\right], \\
\tilde{D}_{I}=\left[\begin{array}{ccc}
D_{I} & \mathbf{0} & \mathbf{0} \\
\mathbf{0} & D_{I_{11}} & \mathbf{0} \\
\mathbf{0} & \mathbf{0} & D_{I_{12}}
\end{array}\right] .
\end{gathered}
$$

The new Fisher Information Matrix is given by,

$$
\tilde{F}=\left[\begin{array}{cc}
\tilde{E}_{1}^{T} \tilde{D}_{R}^{2} \tilde{E}_{1} & \tilde{E}_{1}^{T} \tilde{D}_{R} \tilde{D}_{I} \tilde{E}_{1} \\
\tilde{E}_{1}^{T} \tilde{D}_{R} \tilde{D}_{I} \tilde{E}_{1} & \tilde{E}_{1}^{T} \tilde{D}_{I}^{2} \tilde{E}_{1}
\end{array}\right] .
$$

The individual terms of $\tilde{F}$ can be simplified as shown in $(2)-(5)$ (lengthy equations are in the last page).

Lets now consider adding one more agent to the existing set of agents i.e. $N^{\prime}=2$. The second agent gets measurements from the first $N$ agents and $M$ anchors. In this case we get the following updated matrices,

$$
\begin{aligned}
& \tilde{E}_{1}=\left[\begin{array}{ccc}
E_{1} & \mathbf{0}_{L} & \mathbf{0}_{L} \\
-I_{N} & \mathbf{1}_{N} & \mathbf{0}_{N} \\
\mathbf{0}_{N} & \mathbf{1}_{M} & \mathbf{0}_{M} \\
-I_{N} & \mathbf{0}_{N} & \mathbf{1}_{N} \\
\mathbf{0}_{N} & \mathbf{0}_{M} & \mathbf{1}_{M}
\end{array}\right] \\
& \tilde{D}_{R}=\left[\begin{array}{ccccc}
D_{R} & \mathbf{0} & \mathbf{0} & \mathbf{0} & \mathbf{0} \\
\mathbf{0} & D_{R_{11}} & \mathbf{0} & \mathbf{0} & \mathbf{0} \\
\mathbf{0} & \mathbf{0} & D_{R_{12}} & \mathbf{0} & \mathbf{0} \\
\mathbf{0} & \mathbf{0} & \mathbf{0} & D_{R_{21}} & \mathbf{0} \\
\mathbf{0} & \mathbf{0} & \mathbf{0} & \mathbf{0} & D_{R_{22}}
\end{array}\right]
\end{aligned}
$$

The terms in the new Fisher Information Matrix can be simplified and have the structure shown in (6). The Fisher matrix evolution as more and more nodes are added is apparent from the expression (6). Similar evolution holds for other block terms in the Fisher matrix. To simplify the analysis it would be good if we could separate out the original Fisher Information Matrix terms and express $\tilde{F}$ in terms of $F$. This requires rearranging some of the terms in $\tilde{F}$. Recall the definition of $F$. We had $\eta=\left[\mathbf{u}_{\mathbf{R}}^{\mathbf{T}} \mathbf{u}_{\mathbf{I}}^{\mathbf{T}}\right]^{\mathbf{T}}$, where $\mathbf{u}_{\mathbf{R}}=\operatorname{Re}\{\mathbf{u}\}, \mathbf{u}_{\mathbf{I}}=\operatorname{Im}\{\mathbf{u}\}$. Then $F$ is given by,

$$
F_{i j} \triangleq E\left\{\frac{\partial f(\hat{\mathbf{d}} \mid \eta)}{\partial \eta_{i}} \frac{\partial f(\hat{\mathbf{d}} \mid \eta)}{\partial \eta_{j}}\right\} .
$$


Let $\mathbf{z}=\mathbf{z}_{\mathbf{R}}+\mathbf{j} \mathbf{z}_{\mathbf{I}} \in \mathbb{C}^{N^{\prime} \times 1}$, denote the location of the newly added nodes. The Fisher Information Matrix, $\tilde{F}$ that we have calculated corresponds to the following ordering of the parameters

$$
\tilde{\eta}=\left[\left[\begin{array}{l}
u_{R} \\
z_{R}
\end{array}\right]^{\mathbf{T}}\left[\begin{array}{l}
u_{I} \\
z_{I}
\end{array}\right]^{\mathbf{T}}\right]^{\mathbf{T}}
$$

We will now rearrange the parameters so as to get

$$
\tilde{\tilde{\eta}}=\left[\begin{array}{c}
u_{R} \\
u_{I} \\
z_{R} \\
z_{I}
\end{array}\right] .
$$

Retaining the same notation for $\tilde{F}$, we have the following simplification,

$$
\tilde{F}=\left[\begin{array}{cc}
F+\Delta_{11} & \Delta_{12} \\
\Delta_{21} & \Delta_{22}
\end{array}\right]
$$

where

$$
\Delta_{11}=\left[\begin{array}{cc}
\sum_{j=1}^{N^{\prime}} D_{R_{j 1}}^{2} & \sum_{j=1}^{N^{\prime}} D_{R_{j 1}} D_{I_{j 1}} \\
\sum_{j=1}^{N^{\prime}} D_{R_{j 1}} D_{I_{j 1}} & \sum_{j=1}^{N^{\prime}} D_{I_{j 1}}^{2}
\end{array}\right]
$$

$\Delta_{22}$ and $\Delta_{12}$ are given by the expressions (7) and (9) respectively.

We are now interested in the error improvement of the first $N$ agents after the addition of $N^{\prime}$ agents. For this its sufficient to look at the Schur Complement of the matrix $\Delta_{22}$, since the inverse of the Schur Complement corresponds to the CRLB restricted to the first $N$ nodes. The Schur Complement is given by,

$$
F+\Delta_{11}-\Delta_{12} \Delta_{22}^{-1} \Delta_{21}
$$

Based on similar arguments as in the case of anchor nodes, assuming that each of the newly added nodes are thrown uniform i.i.d into the network, we have for large $N^{\prime}$,

$$
\Delta_{11} \rightarrow \frac{N^{\prime}}{2} I_{2 N}
$$

Let us now assume that the initial set of nodes were also placed randomly and uniformly in the field. Consider the terms in $\Delta_{22}$. Each of the terms $\mathbf{1}_{N}^{T} D_{R_{j 1}}^{2} \mathbf{1}_{N}$ are the sum of the cosine of the angles made by the newly introduced $j$ th node with all the existing nodes in the network. Under the random placement assumption, these angles can also be taken to be distributed i.i.d $U(0,2 \pi)$. Thus we have for large $N$,

$$
\Delta_{22} \rightarrow \frac{N+M}{2} I_{2 N^{\prime}}
$$

Note the difference in this approach as compared to that for the anchor nodes. Here we are averaging over all initial node placements also for this approximation to hold. For the anchor nodes, the result was true for any initial node placement.

With the above approximation in place, we have

$$
\Delta_{12} \Delta_{22}^{-1} \Delta_{12}^{T}=\frac{2}{N+M} \Delta_{12} \Delta_{12}^{T} .
$$

This can be expanded to obtain the expression (10). With the usual law of large numbers argument, each of the terms in the matrix converge as $N^{\prime}$ grows large. The corresponding values to which the $k l$ th term in the matrix converges are shown in $(12)-(18)$. Thus we get,

$$
\Delta_{12} \Delta_{12}^{T} \rightarrow \frac{N^{\prime}}{4}\left(\left[\begin{array}{cc}
\mathbf{1}_{N} \mathbf{1}_{N}^{T} & \mathbf{0} \\
\mathbf{0} & \mathbf{1}_{N} \mathbf{1}_{N}^{T}
\end{array}\right]+I_{2 N}\right)
$$

The Schur complement can now be simplified as shown in (19). The CRLB restricted to the first $N$ nodes is given by the inverse of this Schur Complement. Now consider the case when measurements are obtained only between nodes that are within a radius $R$ of each other. Let $\rho=\frac{\pi R^{2}}{A}$, then similar arguments would simplify the new Fisher matrix $F^{\prime}$ to be,

$$
F+\frac{\rho N^{\prime}}{2}\left(\left(1-\frac{1}{\rho(N+M)}\right) I_{2 N}-\frac{1}{\rho(N+M)}\left[\begin{array}{cc}
\mathbf{1}_{N} \mathbf{1}_{N}^{T} & \mathbf{0} \\
\mathbf{0} & \mathbf{1}_{N} \mathbf{1}_{N}^{T}
\end{array}\right]\right)
$$




$$
\begin{aligned}
& \tilde{E}_{1}^{T} \tilde{D}_{R}^{2} \tilde{E}_{1}=\left[\begin{array}{ccc}
E_{1}^{T} & -I_{N} & \mathbf{0}_{N}^{T} \\
\mathbf{0}_{L}^{T} & \mathbf{1}_{N}^{T} & \mathbf{1}_{M}^{T}
\end{array}\right]\left[\begin{array}{ccc}
D_{R} & \mathbf{0} & \mathbf{0} \\
\mathbf{0} & D_{R_{11}} & \mathbf{0} \\
\mathbf{0} & \mathbf{0} & D_{R_{12}}
\end{array}\right]\left[\begin{array}{cc}
E_{1} & \mathbf{0}_{L} \\
-I_{N} & \mathbf{1}_{N} \\
\mathbf{0}_{N} & \mathbf{1}_{M}
\end{array}\right] \\
& =\left[\begin{array}{cc}
E_{1}^{T} D_{R}^{2} E_{1}+D_{R_{11}}^{2} & -D_{R_{11}}^{2} \mathbf{1}_{N} \\
-\mathbf{1}_{N}^{T} D_{R_{11}}^{2} & \mathbf{1}_{N}^{T} D_{R_{11}}^{2} \mathbf{1}_{N}+\mathbf{1}_{M}^{T} D_{R_{12}}^{2} \mathbf{1}_{M}
\end{array}\right] \\
& \tilde{E}_{1}^{T} \tilde{D}_{I}^{2} \tilde{E}_{1}=\left[\begin{array}{cc}
E_{1}^{T} D_{I}^{2} E_{1}+D_{I_{11}}^{2} & -D_{I_{11}}^{2} \mathbf{1}_{N} \\
-\mathbf{1}_{N}^{T} D_{I_{11}}^{2} & \mathbf{1}_{N}^{T} D_{I_{11}}^{2} \mathbf{1}_{N}+\mathbf{1}_{M}^{T} D_{I_{12}}^{2} \mathbf{1}_{M}
\end{array}\right] \\
& \tilde{E}_{1}^{T} \tilde{D}_{R} \tilde{D}_{I} \tilde{E}_{1}=\left[\begin{array}{cc}
E_{1}^{T} D_{R} D_{I} E_{1}+D_{R_{11}} D_{I_{11}} & -D_{R_{11}} D_{I_{11}} \mathbf{1}_{N} \\
-\mathbf{1}_{N}^{T} D_{R_{11}} D_{I_{11}} & \mathbf{1}_{N}^{T} D_{R_{11}} D_{I_{11}} \mathbf{1}_{N}+\mathbf{1}_{M}^{T} D_{R_{11}} D_{I_{11}} \mathbf{1}_{M}
\end{array}\right] \\
& \tilde{E}_{1}^{T} \tilde{D}_{R}^{2} \tilde{E}_{1}=\left[\begin{array}{ccccc}
E_{1}^{T} & -I_{N} & \mathbf{0}_{N}^{T} & -I_{N} & \mathbf{0}_{N}^{T} \\
\mathbf{0}_{L}^{T} & \mathbf{1}_{N}^{T} & \mathbf{1}_{M}^{T} & \mathbf{0}_{N}^{T} & \mathbf{0}_{M}^{T} \\
\mathbf{0}_{L}^{T} & \mathbf{0}_{N}^{T} & \mathbf{0}_{M}^{T} & \mathbf{1}_{N}^{T} & \mathbf{1}_{M}^{T}
\end{array}\right]\left[\begin{array}{ccccc}
D_{R} & \mathbf{0} & \mathbf{0} & \mathbf{0} & \mathbf{0} \\
\mathbf{0} & D_{R_{11}} & \mathbf{0} & \mathbf{0} & \mathbf{0} \\
\mathbf{0} & \mathbf{0} & D_{R_{12}} & \mathbf{0} & \mathbf{0} \\
\mathbf{0} & \mathbf{0} & \mathbf{0} & D_{R_{21}} & \mathbf{0} \\
\mathbf{0} & \mathbf{0} & \mathbf{0} & \mathbf{0} & D_{R_{22}}
\end{array}\right]\left[\begin{array}{cccc}
E_{1} & \mathbf{0}_{L} & \mathbf{0}_{L} \\
-I_{N} & \mathbf{1}_{N} & \mathbf{0}_{N} \\
\mathbf{0}_{N} & \mathbf{1}_{M} & \mathbf{0}_{M} \\
-I_{N} & \mathbf{0}_{N} & \mathbf{1}_{N} \\
\mathbf{0}_{N} & \mathbf{0}_{M} & \mathbf{1}_{M}
\end{array}\right] \\
& =\left[\begin{array}{ccc}
E_{1}^{T} D_{R}^{2} E_{1}+D_{R_{11}}^{2}+D_{R_{21}}^{2} & -D_{R_{11}}^{2} \mathbf{1}_{N} & -D_{R_{21}}^{2} \mathbf{1}_{N} \\
-\mathbf{1}_{N}^{T} D_{R_{11}}^{2} & \mathbf{1}_{N}^{T} D_{R_{11}}^{2} \mathbf{1}_{N}+\mathbf{1}_{M}^{T} D_{R_{12}}^{2} \mathbf{1}_{M} & \mathbf{0} \\
-\mathbf{1}_{N}^{T} D_{R_{21}}^{2} & \mathbf{0} & \mathbf{1}_{N}^{T} D_{R_{21}}^{2} \mathbf{1}_{N}+\mathbf{1}_{M}^{T} D_{R_{22}}^{2} \mathbf{1}_{M}
\end{array}\right]
\end{aligned}
$$

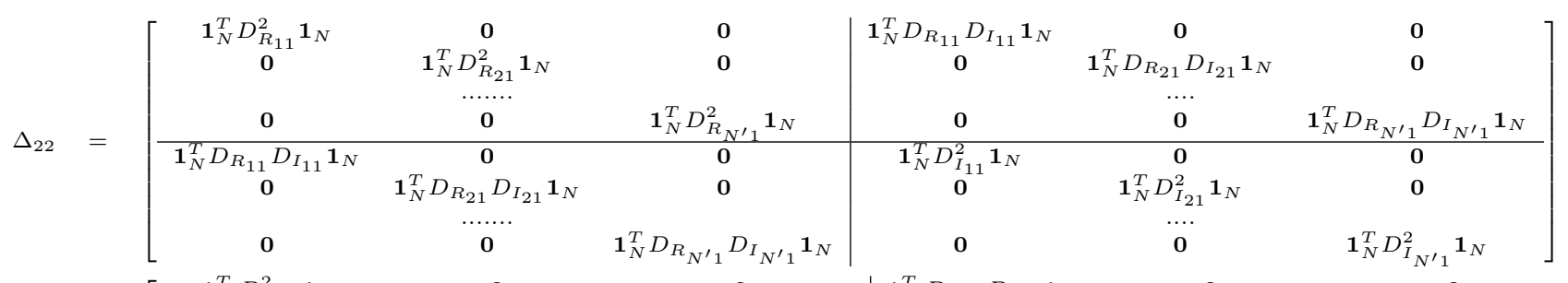

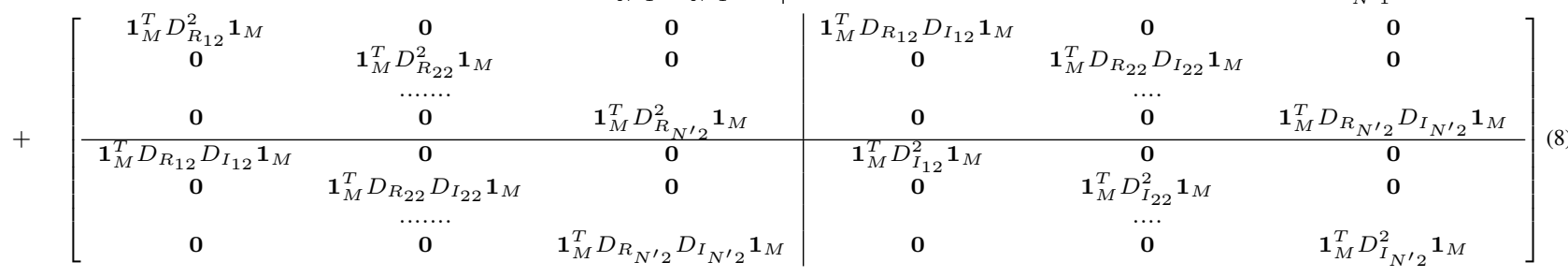

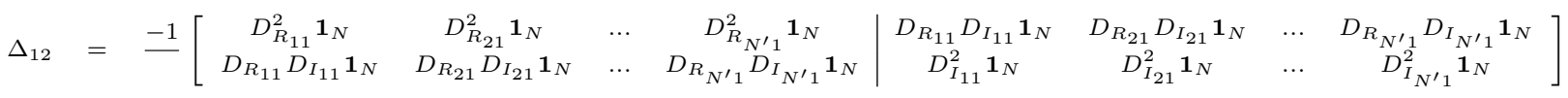

$$
\begin{aligned}
& \Delta_{12} \Delta_{12}^{T}=\left[\begin{array}{c|c|c}
\sum_{j=1}^{N^{\prime}} D_{R_{j 1}}^{2} \mathbf{1}_{N} \mathbf{1}_{N}^{T} D_{R_{j 1}}^{2}+D_{R_{j 1}} D_{I_{j 1}} \mathbf{1}_{N} \mathbf{1}_{N}^{T} D_{R_{j 1}} D_{I_{j 1}} & \sum_{j=1}^{N^{\prime}} D_{R_{j 1}}^{2} \mathbf{1}_{N} \mathbf{1}_{N}^{T} D_{R_{j 1}} D_{I_{j 1}}+D_{R_{j 1}} D_{I_{j 1}} \mathbf{1}_{N} \mathbf{1}_{N}^{T} D_{I_{j 1}}^{2} \\
\sum_{j=1}^{N^{\prime}} D_{R_{j 1}}^{2} \mathbf{1}_{N} \mathbf{1}_{N}^{T} D_{R_{j 1}} D_{I_{j 1}}+D_{R_{j 1}} D_{I_{j 1}} \mathbf{1}_{N} \mathbf{1}_{N}^{T} D_{I_{j 1}}^{2} & \sum_{j=1}^{N^{\prime}} D_{I_{j 1}}^{2} \mathbf{1}_{N} \mathbf{1}_{N}^{T} D_{I_{j 1}}^{2}+D_{R_{j 1}} D_{I_{j 1}} \mathbf{1}_{N} \mathbf{1}_{N}^{T} D_{R_{j 1}} D_{I_{j 1}}
\end{array}\right] \\
& \left(\sum_{j=1}^{N^{\prime}} D_{R_{j 1}}^{2} \mathbf{1}_{N} \mathbf{1}_{N}^{T} D_{R_{j 1}}^{2}\right)(k l)=\sum_{j=1}^{N^{\prime}} \cos \left(\phi_{j}(k)\right)^{2} \cos \left(\phi_{j}(l)\right)^{2} \\
& \rightarrow\left\{\begin{array}{cc}
\frac{3 N^{\prime}}{8} & \text { if } k=l \\
\frac{N^{\prime}}{4} & \text { o.w }
\end{array}\right. \\
& \left(\sum_{j=1}^{N^{\prime}} D_{R_{j 1}}^{2} \mathbf{1}_{N} \mathbf{1}_{N}^{T} D_{R_{j 1}} D_{I_{j 1}}+D_{R_{j 1}} D_{I_{j}} \mathbf{1}_{N} \mathbf{1}_{N}^{T} D_{I_{j 1}}^{2}\right)(k l)=\sum_{j=1}^{N^{\prime}}\left(\cos \left(\phi_{j}(k)\right)^{2} \cos \left(\phi_{j}(l)\right) \sin \left(\phi_{j}(l)\right)+\cos \left(\phi_{j}(k)\right) \sin \left(\phi_{j}(k)\right) \sin \left(\phi_{j}(l)\right)^{2}\right) \\
& \rightarrow 0 \\
& \left.\left.\left(\sum_{j=1}^{N^{\prime}} D_{R_{j 1}} D_{I_{j 1}} \mathbf{1}_{N} \mathbf{1}_{N}^{T} D_{R_{j 1}} D_{I_{j 1}}\right)(k l)=\frac{1}{4} \sum_{j=1}^{N^{\prime}} \sin \phi_{j}(l)\right) \sin \phi_{j}(k)\right) \\
& \rightarrow\left\{\begin{array}{cc}
\frac{N^{\prime}}{8} & \text { if } j=k \\
0 & \text { o.w }
\end{array}\right. \\
& \left(\sum_{j=1}^{N^{\prime}} D_{R_{j 1}} D_{I_{j 1}} \mathbf{1}_{N} \mathbf{1}_{N}^{T} D_{I_{j 1}}^{2}\right)(k l) \rightarrow 0 \\
& F+\Delta_{11}-\Delta_{12} \Delta_{22}^{-1} \Delta_{21} \rightarrow F+\frac{N^{\prime}}{2}\left(\left(1-\frac{1}{N+M}\right) I_{2 N}-\frac{1}{N+M}\left[\begin{array}{cc}
\mathbf{1}_{N} \mathbf{1}_{N}^{T} & \mathbf{0} \\
\mathbf{0} & \mathbf{1}_{N} \mathbf{1}_{N}^{T}
\end{array}\right]\right)
\end{aligned}
$$

